points, into the edge $x y$ of $B$. Then $T$ is an interior transformation. In fact $T$ is a local homeomorphism which is 2 to 1 .

Since $A$ is a planar graph, whereas $B$ is non-planar ( $B$ is, in fact, one of the two well known Kuratowski primitive skew curves), clearly $A$ contains no subset homeomorphic with $B$. Incidentally this example shows that planarity is not an interior property (that is, it is not invariant under interior transformations).

UNIVERSITY OF VIRGINIA

\title{
ON THE TRANSFORMATION GROUP FOR DIABOLIC MAGIC SQUARES OF ORDER FOUR*
}

\section{BARKLEY ROSSER AND R. J. WALKER}

This paper concerns only magic squares of order four, and all statements of the paper are to be construed as applying only to magic squares of order four.

One says that

\begin{tabular}{|c|c|c|c|}
\hline$a$ & $b$ & $c$ & $d$ \\
\hline$e$ & $f$ & $g$ & $h$ \\
\hline$i$ & $j$ & $k$ & $l$ \\
\hline$m$ & $n$ & $o$ & $p$ \\
\hline
\end{tabular}

is a diabolic (or pan-diagonal or Nasik) magic square if $a, b, \cdots, p$ are $1,2, \cdots, 16$ in some order, and each row, column, and diagonal adds up to 34 . This is to include broken diagonals such as $i, f, c, p$, or $c, h, i, n$. A diabolic magic square clearly remains diabolic if subjected to the following transformations:

$A$. Reflection about the $a, f, k, p$ diagonal.

$B$. Rotation through $90^{\circ}$ counter-clockwise.

C. Putting the first column last.

D. Putting the first row last.

For many purposes it is convenient to consider a diabolic magic

* Presented to the Society, December 30, 1937. 
square as mapped on a torus. That is, the square is bent and stretched until the top and bottom edges coincide and the right and left edges coincide. Then a diagonal like $b, g, l, m$ is continuous and not broken.

THEOREM 1. The four elements of any square of order two of a diabolic magic square of order four mapped on a torus add up to 34.

This includes squares like $a, d, m, p$ or $e, h, i, l$.

Proof. Add together the two main diagonals and the second and third rows and subtract the first and fourth columns. This gives $f+g+j+k=34$. By use of transformations $C$ and $D$, this proof can be applied to any square of order two.

THEOREM 2. If a diabolic magic square of order four be mapped on a torus, then the sum of any element and the element which is two distant from it along a diagonal is 17.

Note that if one starts at any element and moves two elements along any diagonal, one always stops at the same element regardless of the direction of the diagonal. Two elements so situated will here be called antipodal.

Proof. Add together the first and the third rows, then add the first and third columns. and the diagonals $a, f, k, p$ and $a, n, k, h$; and subtract the diagonals $e, b, o, l ; i, f, c ; p ; m, j, g, d$; and $i, n, c, h$. This gives $a+k=17$. By use of transformations $C$ and $D$, this proof can be applied to any pair of antipodal elements.

By use of these two theorems, one can readily see that a diabolic magic square remains diabolic if subjected to the following transformation:

$E$. The transformation of (1) into

\begin{tabular}{|c|c|c|c|}
\hline$a$ & $d$ & $h$ & $e$ \\
\hline$b$ & $c$ & $g$ & $f$ \\
\hline$n$ & $o$ & $k$ & $j$ \\
\hline$m$ & $p$ & $l$ & $i$ \\
\hline
\end{tabular}

THEOREM 3. All diabolic magic squares of order four can be derived from a single one by successive applications of $A, B, C, D$, and $E$. 
Proof. By use of $C$ and $D, 1$ can be brought into the upper lefthand corner. Thus there is no loss of generality in taking $a=1, k=16$. Then $c$ and $d$ can at most be 14 and 15 , so that $c+d \leqq 29$. Hence $b \geqq 4$. Similarly $\xi \geqq 4, d \geqq 4, e \geqq 4, i \geqq 4, \quad m \geqq 4$. Since $a+b+e+f=34$, $a+d+e+h=34, a+b+m+n=34$, and $a+d+m+p=34$ by Theorem 1 , one can similarly get $f \geqq 4, h \geqq 4, n \geqq 4, p \geqq 4$. Thus 2 and 3 can only occur in the positions $g, j, l$, and $o$. If 2 occurs in the $o$ or $l$ position, it can be brought to the $j$ position by one or two applications of $E$. If 2 occurs in the $j$ position, it can be brought to the $g$ position by an application of $A$. So one can take $g=2, m=15$ with no loss of generality. If 3 occurs in the $l$ or $j$ position, it can be brought to the $o$ position by one or two applications of $E$. Hence one takes $o=3$, $e=14, i=4, c=13$. Now $d=20-b$, and by Theorem $2, l=17-b$, $j=b-3$. By Theorem $1, f=19-b$, so that $h=b-1, n=18-b$, $p=b-2$. Hence we have to find values of $b$ such that $b-3, b-2$, $b-1, b, 17-b, 18-b, 19-b$, and $20-b$ are $5,6,7,8,9,10,11$, and 12 in some order. Clearly $b=8$ or $b=12$. With $b=8$, we get

\begin{tabular}{|c|c|c|c|}
\hline 1 & 8 & 13 & 12 \\
\hline 14 & 11 & 2 & 7 \\
\hline 4 & 5 & 16 & 9 \\
\hline 15 & 10 & 3 & 6 \\
\hline
\end{tabular}

By applying $C, B$, and $A$ successively to this we get the square that would result from putting $b=12$. Thus all diabolic magic squares can be obtained from the one shown by successive applications of $A, B$, $C, D$, and $E$.

THEOREM 4. There are 384 diabolic magic squares of order four.

Proof. If $X$ and $Y$ are two transformations, denote by $X Y$ the transformation effected by first performing $Y$ and then performing $X$. Then (a) $B A=A B^{3}$; (b) $C A=A D$; (c) $D A=A C$; (d) $C B=B D$; (e) $D B=B C^{3}$; (f) $D C=C D$; (g) $E A=A B^{2} C D E$; (h) $E^{2} A=B^{2} C D E^{2}$; (i) $E B=B^{3} C D E$; (j) $E^{2} B=A B C D E^{2}$; (k) $E C=C^{3} E^{2}$; (l) $E^{2} C$ $=B^{2} C D^{2} E$; (m) $E D=A C E^{2} ;$ (n) $E^{2} D=A B^{2} D E$; (o) $A^{2}=B^{4}=C^{4}$ $=D^{4}=E^{3}=$ the identical transformation. These relations were obtained by inspection and can be easily verified. By means of (a)-(o) one can, in any product of $A, B, C, D, E$, get all the $E$ 's to the right, 
then all $A$ 's to the left, then all $B$ 's next to the $A$ 's, and finally all $C$ 's next to the $B$ 's; so that any product of $A, B, C, D, E$ is equal to a product of the form $A^{\alpha} B^{\beta} C^{\gamma} D^{\delta} E^{\epsilon}$. Moreover $A, B, C$, and $D$ all carry rows into rows and columns into columns, or rows into columns and columns into rows, so that neither $E$ nor $E^{2}$ is a product of $A, B, C$, and $D$. Moreover, if a square is mapped on a torus, $B, C$, and $D$ all leave the orientation unchanged, so that $A$ is not a product of $B, C$, and $D$. Clearly $B, C$, and $D$ are independent. Hence $A^{\alpha} B^{\beta} C^{\gamma} D^{\delta} E^{\epsilon}$ $=A^{a} B^{b} C^{c} D^{d} E^{e}$ if and only if $\alpha \equiv a(2), \beta \equiv b(4), \gamma \equiv c(4), \delta \equiv d(4)$, and $\epsilon \equiv e(3)$. Hence the order of the group generated by $A, B, C, D$, and $E$ is $2 \times 4 \times 4 \times 4 \times 3=384$. ${ }^{*}$ As each transformation is a permutation of the sixteen elements of the square, different transformations cannot yield the same square. So there are 384 diabolic magic squares.

This theorem disagrees with the result of $\mathrm{D}$. N. Lehmer, $A$ census of squares of order 4 , magic in rows, columns, and diagonals, this Bulletin, vol. 39 (1933), p. 981. Lehmer's count was 48. It is easy to see the source of Lehmer's error. Lehmer found all squares which were normalized with respect to certain transformations, $R, S, T$, and $U$, and picked out the diabolic ones. However, Lehmer's transformation $U$ destroys diabolism, thus certain of his normalized squares which are not diabolic give rise to diabolic squares when operated on by $U$. Hence Lehmer missed these squares in his count.

The present count was checked against Frenicle's list of magic squares. In so doing, we discovered the following startling result:

If there are two antipodal elements of a magic square of order four which add up to 17 , then the magic square is diabolic.

The defining conditions of a magic square plus the single condition that a particular pair of antipodal elements add up to 17 give ten independent equations in the sixteen elements of the square, as contrasted with twelve independent equations arising from the defining conditions for a diabolic magic square. That the two sets of equations are equivalent is due to the diophantine condition that the elements must be $1,2, \cdots, 16$ in some order.

${ }^{*}$ H. S. M. Coxeter has suggested that this group may be isomorphic with the symmetry group of the Cartesian frame in four dimensions, as the two groups are of the same order. That this is so can be shown as follows. The elements $R_{1}=A B C E$, $R_{2}=A B^{3} D, R_{3}=B C E, R_{4}=A B$, will generate our group, since $A=R_{1} R_{3}, B=R_{1} R_{3} R_{4}$, $C=R_{4} R_{3} R_{1} R_{2} R_{1} R_{3}, D=R_{3} R_{4} R_{3} R_{2}, E=R_{1} R_{3} R_{2} R_{3}$, as may be verified by the relations (a)-(o). The $R_{i}$ satisfy the relations $R_{i}^{2}=\left(R_{1} R_{3}\right)^{2}=\left(R_{1} R_{4}\right)^{2}=\left(R_{2} R_{4}\right)^{2}=\left(R_{1} R_{2}\right)^{3}$ $=\left(R_{2} R_{3}\right)^{3}=\left(R_{3} R_{4}\right)^{4}=1$, hence our group is a factor group of the above mentioned symmetry group (Coxeter, Journal of the London Mathematical Society, vol. 10 (1935), pp. 21-25). The group in question is designated by $\left[3^{2}, 4\right]$. Since the two groups have the same order they are isomorphic. 
If one weakens the requirements still further and only asks that a square be magic in the rows and columns, then a pair of antipodal elements can add up to 17 without the square being diabolic. This is illustrated by

\begin{tabular}{|r|r|r|r|}
\hline 1 & 15 & 4 & 14 \\
\hline 12 & 2 & 9 & 11 \\
\hline 8 & 7 & 16 & 3 \\
\hline 13 & 10 & 5 & 6 \\
\hline
\end{tabular}

which is magic in rows and columns, but not in diagonals, and which has $a+k=e+o=17$.

An analogous treatment of the problem of finding all diabolic magic squares is given by Kraitchik on page 167 of his book, La Mathématique des Jeux, where he shows that all diabolic magic squares can be derived by successive applications of $A, B, C$, and $D$ from three particular ones which he gives.

Cornell University

\section{A NOTE ON REGULAR BANACH SPACES*}

\section{B. J. PETTIS}

Introduction. For an element $x$ of a Banach space $B_{0} \dagger$ it is well known that the functional

$$
X_{x}(f)=f(x)
$$

defined over $B_{1}=\bar{B}_{0}$, the Banach space composed of all linear functionals (real-valued additive and continuous functions) defined over $B_{0}$, is linear; moreover $\ddagger$

$$
\left\|X_{x}\right\|_{\bar{B}_{1}}=\|x\|_{B_{0}} ;
$$

hence the additive operation $X_{x}=T(x)$ from $B_{0}$ to $B_{2}=\bar{B}_{1}$ is continuous and norm-preserving. In $B_{2}$ let $B_{2}{ }^{(0)}$ denote the set of image ele-

* Presented to the Society, October 30, 1937.

$\dagger$ S. Banach, Théorie des Opérations Linéaires, Warsaw, 1932, p. 53. We shall use Banach's terminology.

$\ddagger$ Banach, loc. cit., pp. 188-189. 